TRANSACTIONS OF THE

AMERICAN MATHEMATICAL SOCIETY

Volume 350, Number 6, June 1998, Pages 2277-2296

S 0002-9947(98)01835-2

\title{
WEAK*-CLOSEDNESS OF SUBSPACES OF FOURIER-STIELTJES ALGEBRAS AND WEAK*-CONTINUITY OF THE RESTRICTION MAP
}

\author{
M. B. BEKKA, E. KANIUTH, A. T. LAU, AND G. SCHLICHTING
}

Dedicated to Professor Elmar Thoma on the occasion of his seventieth birthday

\begin{abstract}
Let $G$ be a locally compact group and $B(G)$ the Fourier-Stieltjes algebra of $G$. We study the problem of how weak*-closedness of some translation invariant subspaces of $B(G)$ is related to the structure of $G$. Moreover, we prove that for a closed subgroup $H$ of $G$, the restriction map from $B(G)$ to $B(H)$ is weak*-continuous only when $H$ is open in $G$.
\end{abstract}

\section{INTRODUCTION}

Let $G$ be a locally compact group, and let $B(G)$ be the Fourier-Stieltjes algebra of $G$ as defined by Eymard [8]. Recall that $B(G)$ is the linear span of all continuous positive definite functions on $G$ and can be identified with the Banach space dual of $C^{*}(G)$, the group $C^{*}$-algebra of $G$. The space $B(G)$, with the norm as dual of $C^{*}(G)$, is a commutative Banach *-algebra with pointwise multiplication and complex conjugation. The Fourier algebra $A(G)$ of $G$ is the closed *-subalgebra of $B(G)$ generated by the functions in $B(G)$ with compact support. In particular, $A(G)$ is contained in $C_{0}(G)$, the algebra of complex valued continuous functions on $G$ vanishing at infinity. As is well known $A(G)$ is weak*-dense in $B(G)$ if and only if $G$ is amenable. In [3] translation invariant *-subalgebras $A$ of $B(G)$ were studied, and it was shown that if such $A$ is weak*-closed and point separating, then it must contain $A(G)$. However, apart from this, very little seems to be known about weak*-closed subspaces of $B(G)$.

The first purpose of this paper is to investigate the relation between weak*closedness of certain interesting norm-closed translation invariant subspaces of $B(G)$ and the structure of $G$. Secondly, we solve the problem of when, for a closed subgroup $H$ of $G$, the restriction map from $B(G)$ to $B(H)$ is weak*-continuous.

A brief outline of the paper is as follows. In Section 2 we establish for almost connected locally compact groups $G$ the relation between weak*-closedness of $B_{0}(G)=B(G) \cap C_{0}(G)$ in $B(G)$ and the structure of $G$ (Theorem 2.10). The key result is that for a connected Lie group $G, B_{0}(G)$ is weak*-closed in $B(G)$ if and only if $G$ is a reductive Lie group with compact centre and Kazhdan's property $(T)$.

Received by the editors December 15, 1995.

1991 Mathematics Subject Classification. Primary 22D10, 43A30.

Work supported by NATO collaborative research grant CRG 940184 .

(C)1997 American Mathematical Society 
If $G$ is a compact group, then for any unitary representation $\pi$ of $G$, the Fourier space $A_{\pi}(G)$ associated to $\pi$ (see [1] for the definition and properties of $A_{\pi}(G)$ ) is weak*-closed in $B(G)$. Note that $A(G)=A_{\lambda_{G}}(G)$, where $\lambda_{G}$ denotes the left regular representation of $G$. In Theorem 3.6 we shall show that, conversely, if $G$ contains an almost connected open normal subgroup and $A(G)$ is weak*-closed in $B(G)$, then $G$ is compact. We also give a characterization of compactness of $G$ in terms of the weak* and the norm topologies on the unit sphere of $B(G)$ (Theorem $3.9)$.

Besides the left regular representation, one of the most interesting representations of a locally compact group $G$ is the conjugation representation $\gamma_{G}$ of $G$ on $L^{2}(G)$. In contrast to $A(G), A_{\gamma_{G}}(G)$ need not be a subalgebra and it can at best determine the structure of $G / Z(G)$, where $Z(G)$ denotes the centre of $G$. We prove that if $G$ is a Lie group with countably many connected components and $A_{\gamma_{G}}(G)$ is weak*-closed in $B(G)$, then $G / Z(G)$ is compact (Theorem 4.8).

Let $H$ be a closed subgroup of an arbitrary locally compact group $G$. Clearly, the restriction map $B(G) \rightarrow B(H)$ is continuous for the weak*-topologies whenever $H$ is open. In the final section 5 we succeed in showing that conversely weak*-continuity of the restriction map forces $H$ to be open in $G$.

\section{Preliminaries}

Throughout this paper, $G$ denotes a locally compact group with a fixed left Haar measure $d x$ and modular function $\Delta, L^{1}(G)$ the convolution algebra of integrable functions on $G$ and $C^{*}(G)$ the group $C^{*}$-algebra of $G$. The Fourier-Stieltjes algebra $B(G)$ is the Banach space dual of $C^{*}(G)$ and as such carries the weak*-topology ( $w^{*}$ topology, for short) $\sigma\left(B(G), C^{*}(G)\right)$. The basic reference on Fourier and FourierStieltjes algebras is [8].

Next, we have to introduce some notation from representation theory. We use the same letter, for example $\pi$, for a unitary representation of $G$ and for the corresponding *-representation of $C^{*}(G) . \mathcal{H}_{\pi}$ will always denote the Hilbert space of $\pi$ and ker $\pi$ the $C^{*}$-kernel of $\pi$. If $S$ and $T$ are sets of unitary representations of $G$, then $S$ is weakly contained in $T(S \prec T)$ if $\bigcap_{\sigma \in S} \operatorname{ker} \sigma \supseteq \bigcap_{\tau \in T} \operatorname{ker} \tau$ or, equivalently, if every positive definite function associated to $S$ can be uniformly approximated on compact subsets of $G$ by sums of positive definite functions associated to $T$. Also $S$ and $T$ are weakly equivalent $(S \sim T)$ if $S \prec T$ and $T \prec S$.

The dual space $\widehat{G}$ of $G$ is the set of equivalence classes of irreducible representations of $G$, endowed with the Jacobson topology. For a representation $\pi$ of $G$, the support of $\pi$ is the closed subset $\operatorname{supp} \pi=\{\rho \in \widehat{G}: \rho \prec \pi\}$ of $\widehat{G}$. In particular, the support of the left regular representation $\lambda_{G}$ is the reduced dual $\widehat{G}_{r}$, and $\lambda_{G}\left(C^{*}(G)\right)$ is the so-called reduced group $C^{*}$-algebra of $G$ which is denoted by $C_{r}^{*}(G)$. If $N$ is a closed normal subgroup of $G$, then every representation of $G / N$ can be lifted to a representation of $G$, and in this sense, $(G / N)^{\wedge}$ will always be regarded as a subset of $\widehat{G}$. For general references to representation theory and dual spaces we mention [5] and [10].

$G$ is called amenable if there exists a non-zero positive linear functional $m$ on the space $C^{b}(G)$ of bounded continuous complex valued functions on $G$ such that $m\left({ }_{x} f\right)=m(f)$ for all $f \in C^{b}(G)$ and $x \in G$, where ${ }_{x} f(y)=f\left(x^{-1} y\right)$. Recall that amenability is equivalent to a number of different conditions: $C_{r}^{*}(G)=C^{*}(G)$, 
$\widehat{G}_{r}=\widehat{G}$ or $1_{G} \prec \lambda_{G}$ where $1_{G}$ is the trivial one-dimensional representation of $G$. Concerning the theory of amenable groups, we refer the reader to [29] and [30].

Let $H$ be a closed subgroup of $G$, and suppose that $\sigma$ and $\pi$ are representations of $H$ and $G$ respectively. The representation of $G$ induced by $\sigma$ is denoted $\operatorname{ind}_{H}^{G} \sigma$. Then the tensor product $\pi \otimes \operatorname{ind}_{H}^{G} \sigma$ is equivalent to $\operatorname{ind}_{H}^{G}(\pi \mid H \otimes \sigma)$. Furthermore, by the theorem on induction in stages, $\operatorname{ind}_{H}^{G} \sigma=\operatorname{ind}_{K}^{G}\left(\operatorname{ind}_{H}^{K} \sigma\right)$ for every closed subgroup $K$ of $G$ containing $H$. Finally, we will frequently use that $\pi \prec \operatorname{ind}_{H}^{G}(\pi \mid H)$ if $G$ is amenable [12, Theorem 5.1].

Let $A$ be a $C^{*}$-algebra and $\widehat{A}$ its dual space, i.e. the set of equivalence classes of non-degenerated irreducible ${ }^{*}$-representations of $A$. We will several times use the fact that if the Banach space dual $A^{*}$ of $A$ is separable in the norm topology, then $\widehat{A}$ is countable (see [15, Theorem 3.1] or [23, Lemma 4.12]).

\section{When is $B_{0}(G) w^{*}$-Closed IN $B(G)$ ?}

For a locally compact group $G$, let $B_{0}(G)=B(G) \cap C_{0}(G)$ denote the normclosed and translation invariant subalgebra of $B(G)$ consisting of all functions in $B(G)$ that vanish at infinity. In this section we shall study the problem of when $B_{0}(G)$ is closed in $B(G)$ with respect to the $w^{*}$-topology on $B(G)$.

It turns out to be appropriate to reformulate this condition in terms of convergence of positive definite functions. The following lemma, which is a consequence of $[8,(2.1)$ and $(2.12)]$, will be used frequently.

Lemma 2.1. Let $P(G)$ denote the set of all normalized continuous positive definite functions on $G$. The following are equivalent:

(i) $B_{0}(G)$ is $w^{*}$-closed in $B(G)$.

(ii) If $\left(\varphi_{\iota}\right)$ is a net in $P(G) \cap C_{0}(G)$ converging to some $\varphi \in P(G)$ uniformly on compact subsets of $G$, then $\varphi \in C_{0}(G)$.

Lemma 2.2. Suppose that $B_{0}(G)$ is $w^{*}$-closed in $B(G)$. Then every amenable closed normal subgroup of $G$ is compact. In particular, for an amenable group $G$, $B_{0}(G)$ is $w^{*}$-closed in $B(G)$ only when $G$ is compact.

Proof. Let $N$ be an amenable closed normal subgroup of $G$. Then the trivial onedimensional representation $1_{N}$ of $N$ is weakly contained in the left regular representation $\lambda_{N}$ of $N$. Hence, by continuity of inducing, $\operatorname{ind}_{N}^{G} 1_{N}$ is weakly contained in $\operatorname{ind}_{N}^{G} \lambda_{N}$, which is equivalent to $\lambda_{G}$.

Let $q: G \rightarrow G / N$ denote the quotient homomorphism. Then $\operatorname{ind}_{N}^{G} 1_{N}=\lambda_{G / N} \circ q$. Thus every positive definite function $\varphi$ associated to $\lambda_{G / N} \circ q$ is a uniform on compacta limit of functions in $A(G) \cap P(G) \subseteq B_{0}(G)$. Hence, by hypothesis, $\varphi \in C_{0}(G)$. Since such a $\varphi$ is constant on cosets of $N, N$ must be compact.

We continue with two inheritance properties.

Lemma 2.3. Let $H$ be an open subgroup of the locally compact group $G$. If $B_{0}(G)$ is $w^{*}$-closed in $B(G)$, then $B_{0}(H)$ is $w^{*}$-closed in $B(H)$.

Proof. Let $\left(\varphi_{\iota}\right)$ be a net in $P(H) \cap C_{0}(H)$ such that $\varphi_{\iota} \rightarrow \varphi \in P(H)$ uniformly on compact subsets of $H$. Let $\widetilde{\varphi_{\iota}}$ and $\tilde{\varphi}$ denote the trivial extensions of $\varphi_{\iota}$ and $\varphi$ to $G$, that is, $\tilde{\varphi}(x)=\widetilde{\varphi_{\iota}}(x)=0$ for $x \in G \backslash H$. Clearly, then $\widetilde{\varphi_{\iota}} \in P(G) \cap C_{0}(G), \tilde{\varphi} \in P(G)$ and $\widetilde{\varphi_{\iota}} \rightarrow \tilde{\varphi}$ uniformly on compact subsets of $G$. By hypothesis, $\tilde{\varphi} \in C_{0}(G)$ and hence $\varphi \in C_{0}(H)$. 
Lemma 2.4. Let $G$ be a locally compact group and $K$ a compact normal subgroup of $G$. Then $B_{0}(G)$ is $w^{*}$-closed in $B(G)$ if and only if $B_{0}(G / K)$ is $w^{*}$-closed in $B(G / K)$.

Proof. Suppose that $B_{0}(G)$ is $w^{*}$-closed in $B(G)$, and let $\left(\varphi_{\iota}\right)$ be a net in $P(G / K) \cap$ $C_{0}(G / K)$ converging to some $\varphi \in P(G / K)$ uniformly on compact subsets of $G / K$. Then, with $q: G \rightarrow G / K$ the quotient homomorphism, $\varphi_{\iota} \circ q \rightarrow \varphi \circ q$ uniformly on compact subsets of $G$ and $\varphi_{\iota} \circ q \in C_{0}(G)$ since $K$ is comapct. Hence, by hypothesis, $\varphi \circ q \in C_{0}(G)$ and so $\varphi \in C_{0}(G / K)$.

Conversely, suppose that $B_{0}(G / K)$ is $w^{*}$-closed in $B(G / K)$, and let $\varphi \in P(G)$ and $\left(\varphi_{\iota}\right) \subseteq P(G) \cap C_{0}(G)$ such that $\varphi_{\iota} \rightarrow \varphi$ uniformly on compact subsets of $G$. Define $\psi_{\iota}$ and $\psi$ on $G / K$ by

$$
\psi_{\iota}(x K)=\int_{K}\left|\varphi_{\iota}(x k)\right|^{2} d k \text { and } \psi(x K)=\int_{K}|\varphi(x k)|^{2} d k,
$$

$x \in G$ ( $d k$ being the normalized Haar measure on $K)$. Then

$$
\psi \in P(G / K) \text { and } \psi_{\iota} \in P(G / K) \cap C_{0}(G / K),
$$

and $\psi_{\iota} \rightarrow \psi$ uniformly on compact subsets of $G / K$. Hence $\psi \in C_{0}(G / K)$.

For $\delta \in \widehat{K}$, let $\chi_{\delta}$ denote the corresponding minimal idempotent in $L^{1}(K)$. Then by the Cauchy-Schwarz inequality,

$$
\left|\varphi * \chi_{\delta}(x)\right| \leq|\psi(x K)|^{1 / 2}
$$

for every $x \in G$. Since $K$ is compact, this implies $\varphi * \chi_{\delta} \in C_{0}(G)$ for each $\delta \in \widehat{K}$. Now the linear span of $\left\{\chi_{\delta}: \delta \in \widehat{K}\right\}$ is dense in $Z\left(L^{1}(K)\right)$, the centre of $L^{1}(K)$. It follows that for any $f \in Z\left(L^{1}(K)\right), \varphi * f$ is a uniform limit on $G$ of finite linear combinations of functions $\varphi * \chi_{\delta}, \delta \in \widehat{K}$. Hence $\varphi * f \in C_{0}(G)$ for every $f \in Z\left(L^{1}(K)\right)$. Finally, taking for $f$ functions in $Z\left(L^{1}(K)\right)$ with support shrinking to $\{e\}$, we easily conclude that $\varphi \in C_{0}(G)$. This completes the proof.

We now turn to connected Lie groups. Theorem 2.7 below is the key result in this section.

Lemma 2.5. Let $G$ be a connected Lie group and $N$ a connected closed normal subgroup. If $B_{0}(G)$ is $w^{*}$-closed in $B(G)$, then the centre $Z(N)$ of $N$ is compact and $N / Z(N)$ is semisimple.

Proof. Let $R$ denote the radical of $N$. Then $R$ and $Z(N)$ are amenable normal subgroups of $G$, and therefore both must be compact by Lemma 2.2. Since $R$ is solvable and connected Lie, it is isomorphic to a torus $\mathbb{T}^{n}$. Hence $\operatorname{Aut}(R)$, the automorphism group of $R$, is discrete. Now, $G$ acts by conjugation on $R$, and this defines a continuous homomorphism from $G$ into $\operatorname{Aut}(R)$. $G$ being connected, this homomorphism has to be trivial. This shows that $R$ is contained in the centre of $G$. So $R \subseteq Z(N)$ and hence $N / Z(N)$ is semisimple.

We remind the reader that a locally compact group $G$ is said to have Kazhdan's property $(T)$ if the trivial representation $1_{G}$ is an isolated point in the dual space $\widehat{G}$. An amenable group satisfies $(T)$ if and only if it is compact. On the other hand, many connected semisimple Lie groups and many discrete groups share property $(T)$. A comprehensive account on groups with property $(T)$ has been given in [14]. 
Lemma 2.6. Let $G$ be a connected Lie group such that $B_{0}(G)$ is $w^{*}$-closed in $B(G)$. Then $G$ has property $(T)$.

Proof. By Lemma 2.5, $G$ is reductive with compact centre. Let

$$
\widetilde{G}=\mathbb{R}^{n} \times G_{1} \times \cdots \times G_{m}
$$

be the universal covering group of $G$, where $G_{1}, \ldots, G_{m}$ are simply connected Lie groups. Denote by $Z_{i}$ the (discrete) centre of $G_{i}, i=1, \ldots, m$. Then $G=\widetilde{G} / \Gamma$ for some discrete subgroup $\Gamma$ of the centre $Z(\widetilde{G})=\mathbb{R}^{n} \times Z_{1} \times \cdots \times Z_{m}$ of $\widetilde{G}$. Moreover, $Z(G)=Z(\widetilde{G}) / \Gamma$. Hence

$$
G / Z(G)=\widetilde{G} / Z(\widetilde{G})=G_{1} / Z_{1} \times \cdots \times G_{m} / Z_{m} .
$$

Since $Z(G)$ is compact, $B_{0}(G / Z(G))$ is $w^{*}$-closed in $B(G / Z(G)$ ) (Lemma 2.4). Assume, towards a contradiction, that $G$ does not have property $(T)$. Then $G / Z(G)$ does not have property $(T)$. Hence some factor, say $G_{1} / Z_{1}$, fails to have property $(T)$ (see [35, Lemma 7.4.1]). Now, recall the following result due to Howe and Moore [35, Theorem 2.2.20]. If $\pi$ is a unitary representation of a simple Lie group with finite centre and if there are no non-zero $\pi$-invariant vectors, then all the matrix coefficients of $\pi$ vanish at infinity. Therefore, there exists a sequence

$$
\left(\varphi_{n}^{(1)}\right) \subseteq P\left(G_{1} / Z_{1}\right) \cap C_{0}\left(G_{1} / Z_{1}\right)
$$

converging to 1 uniformly on compact subsets of $G_{1} / Z_{1}$. Observe that $G_{1} / Z_{1}$ is not compact. Now choose arbitrary

$$
\varphi^{(k)} \in P\left(G_{k} / Z_{k}\right) \cap C_{0}\left(G_{k} / Z_{k}\right),
$$

$k=2, \ldots, m$, and set

$$
\varphi_{n}=\varphi_{n}^{(1)} \times \varphi^{(2)} \times \cdots \times \varphi^{(m)}
$$

Clearly, $\left(\varphi_{n}\right)$ is a sequence of continuous positive definite functions on $G / Z(G)$ that vanish at infinity, and

$$
\varphi_{n} \rightarrow 1 \times \varphi^{(2)} \times \cdots \times \varphi^{(m)}
$$

uniformly on compact subsets of $G / Z(G)$. Since $1 \times \varphi^{(2)} \times \cdots \times \varphi^{(m)}$ does not vanish at infinity, we have reached a contradiction. Thus $G$ has property $(T)$.

Theorem 2.7. Let $G$ be a connected Lie group. Then $B_{0}(G)$ is $w^{*}$-closed in $B(G)$ if and only if $G$ is a reductive Lie group with compact centre and Kazhdan's property $(T)$.

Proof. From Lemma 2.5, applied to $N=G$, and Lemma 2.6 we know that $w^{*}$ closedness of $B_{0}(G)$ in $B(G)$ implies the stated conditions on $G$.

Suppose now that $G$ is a connected reductive Lie group with compact centre $Z(G)$ and property $(T)$. According to Lemma 2.4 it suffices to show that $B_{0}(G / Z(G))$ is $w^{*}$-closed in $B(G / Z(G))$. Recall from the proof of Lemma 2.6 that $G / Z(G)$ has a decomposition

$$
G / Z(G)=G_{1} / Z_{1} \times \cdots \times G_{m} / Z_{m},
$$

where $G_{1}, \ldots, G_{m}$ are connected simple Lie groups with centres $Z_{1}, \ldots, Z_{m}$, respectively. Set $H=G / Z(G)$ and $H_{i}=G_{i} / Z_{i}$ for $i=1, \ldots, m$, and observe that every $H_{i}$ has property $(T)$. Of course, according to Lemma 2.4 we can assume that none of the $H_{i}$ is compact. 
Let $\varphi_{n} \in P(H) \cap C_{0}(H), n \in \mathbb{N}$, such that $\varphi_{n} \rightarrow \varphi$ uniformly on compact subsets of $G$ for some $\varphi \in P(H)$. Let $\pi_{n}$ and $\pi$ denote the representations of $H$ associated to $\varphi_{n}$ and $\varphi$ through the GNS-construction. Then $\pi$ is weakly contained in the direct sum $\bigoplus_{n=1}^{\infty} \pi_{n}$.

We claim that the restriction $\pi \mid H_{i}$ of $\pi$ to $H_{i}$ does not contain the trivial representation $1_{H_{i}}$. Indeed, otherwise for some $n \in \mathbb{N}, \pi_{n} \mid H_{i}$ contains $1_{H_{i}}$ since $H_{i}$ has property $(T)$. However, since $\varphi_{n} \in C_{0}(H)$, all the matrix coefficients of $\pi_{n}$ vanish at infinity.

We have thus verified that $H$ satisfies the hypotheses of the Howe-Moore theorem [35, Theorem 2.2.20]. It follows that all the matrix coefficients of $\pi$ vanish at infinity. This proves that $\varphi \in C_{0}(H)$.

In order to deal with almost connected groups we need one more lemma.

Lemma 2.8. Let $G$ be a locally compact group and $H$ a closed subgroup such that $G / H$ is compact. If $B_{0}(H)$ is $w^{*}$-closed in $B(H)$, then $B_{0}(G)$ is $w^{*}$-closed in $B(G)$.

Proof. For any function $\phi$ on $G$ and $x, y \in G$, let

$$
{ }_{x} \phi(y)=\phi\left(x^{-1} y\right), \phi_{x}(y)=\phi(y x) \text { and } \phi^{x}(y)=\phi\left(x^{-1} y x\right) .
$$

Notice first that if $\psi$ is a positive definite function on $G$, then $\psi^{x}+\psi+{ }_{x} \psi+\psi_{x}$ and $\psi^{x}+\psi+i\left({ }_{x} \psi-\psi_{x}\right)$ are also positive definite for every $x \in G$. Indeed, for all $f \in L^{1}(G)$

$$
\left\langle\psi^{x}+\psi+{ }_{x} \psi+\psi_{x}, f\right\rangle=\left\langle\psi,\left(\delta_{x}+\delta_{e}\right)^{*} * f *\left(\delta_{x}+\delta_{e}\right)\right\rangle
$$

and

$$
\left\langle\psi^{x}+\psi+i\left({ }_{x} \psi-\psi_{x}\right), f\right\rangle=\left\langle\psi,\left(\delta_{e}-i \delta_{x}\right)^{*} * f *\left(\delta_{e}-i \delta_{x}\right)\right\rangle
$$

Let $\left(\varphi_{\iota}\right)$ be a net in $P(G) \cap C_{0}(G)$ converging to some $\varphi \in P(G)$ uniformly on compact subsets of $G$. Then, uniformly on compact subsets of $G, \varphi_{\iota}^{x} \rightarrow \varphi^{x}$,

$$
\varphi_{\iota}^{x}+\varphi_{\iota}+{ }_{x}\left(\varphi_{\iota}\right)+\left(\varphi_{\iota}\right)_{x} \rightarrow \varphi^{x}+\varphi+{ }_{x} \varphi+\varphi_{x}
$$

and

$$
\varphi_{\iota}^{x}+\varphi_{\iota}+i\left({ }_{x} \varphi_{\iota}-\left(\varphi_{\iota}\right)_{x}\right) \rightarrow \varphi^{x}+\varphi+i\left({ }_{x} \varphi-\varphi_{x}\right)
$$

for every $x \in G$. Thus, since $B_{0}(H)$ is $w^{*}$-closed in $B(H)$,

$$
\varphi\left|H, \varphi^{x}\right| H,\left(\varphi^{x}+\varphi+{ }_{x} \varphi+\varphi_{x}\right) \mid H \text { and }\left(\varphi^{x}+\varphi+i\left({ }_{x} \varphi-\varphi_{x}\right)\right) \mid H
$$

vanish at infinity on $H$. It follows that ${ }_{x} \varphi \mid H \in C_{0}(H)$ for each $x \in G$. Since $G / H$ is compact, employing the uniform continuity of $\varphi$, it is easily verified that $\varphi \in C_{0}(G)$.

The converse to Lemma 2.8 does not hold in general. That is, if $B_{0}(G)$ is $w^{*}$ closed in $B(G)$ and $H$ is a closed cocompact subgroup of $G$, then $B_{0}(H)$ need not be $w^{*}$-closed in $B(H)$. As an example, take for $G$ a simply connected Lie group with finite centre and property $(T)$ and for $H$ a minimal parabolic subgroup. Then by the Howe-Moore result referred to in the proofs of Lemma 2.6 and Theorem 2.7, $B_{0}(G)$ is $w^{*}$-closed in $B(G)$, while $B_{0}(H)$ fails to be $w^{*}$-closed in $B(H)$ since $H$ is non-compact and amenable.

Corollary 2.9. Let $G$ be a connected Lie group and $N$ a connected closed normal subgroup of $G$ such that $G / N$ is compact. Then $B_{0}(G)$ is $w^{*}$-closed in $B(G)$ if and only if $B_{0}(N)$ is $w^{*}$-closed in $B(N)$. 
Proof. Suppose that $B_{0}(G)$ is $w^{*}$-closed in $B(G)$. By Lemma 2.5, $Z(N)$ is compact and $N / Z(N)$ is semisimple. Also, since $G$ has property $(T)$ by Lemma 2.6 and $G / N$ is compact, $N$ has property $(T)$ [34, Theorem 3.7]. By Theorem 2.7 this implies that $B_{0}(N)$ is $w^{*}$-closed in $B(N)$.

The converse is a special case of Lemma 2.8.

Theorem 2.10. Let $G$ be an almost connected locally compact group. Then $B_{0}(G)$ is $w^{*}$-closed in $B(G)$ if and only if the connected component $G_{0}$ of $G$ is a projective limit of reductive Lie groups with property $(T)$ and compact centres.

Proof. Suppose first that $G_{0}$ has the indicated structure. Choose a compact normal subgroup $K$ of $G_{0}$ such that $G_{0} / K$ is a reductive Lie group with property $(T)$ and compact centre. By Theorem 2.7, $B_{0}\left(G_{0} / K\right)$ is $w^{*}$-closed in $B\left(G_{0} / K\right)$. Since $K$ and $G / G_{0}$ are compact, an application of Lemmas 2.4 and 2.8 yields that $B_{0}(G)$ is $w^{*}$-closed in $B(G)$.

Conversely, suppose that $B_{0}(G)$ is $w^{*}$-closed in $B(G)$. $G$ being almost connected it is a projective limit of Lie groups $G / K_{\iota}$. Thus there are closed normal subgroups $H_{\iota}$ of finite index in $G$ such that $K_{\iota} \subseteq H_{\iota}$ and $H_{\iota} / K_{\iota}=\left(G / K_{\iota}\right)_{0}$. Then $G_{0}$ is the projective limit of the groups $G_{0} / G_{0} \cap K_{\iota}$, and the $G_{0} / G_{0} \cap K_{\iota}$ are connected Lie groups since

$$
G_{0} / G_{0} \cap K_{\iota}=G_{0} K_{\iota} / K_{\iota}
$$

a closed connected subgroup of $G / K_{\iota}$. By Theorem 2.7 it suffices to show that $B_{0}\left(G_{0} / G_{0} \cap K_{\iota}\right)$ is $w^{*}$-closed in $B\left(G_{0} / G_{0} \cap K_{\iota}\right)$.

Now, since $B_{0}(G)$ is $w^{*}$-closed in $B(G), B_{0}\left(G / K_{\iota}\right)$ is $w^{*}$-closed in $B\left(G / K_{\iota}\right)$ by Lemma 2.4, and hence $B_{0}\left(H_{\iota} / K_{\iota}\right)$ is $w^{*}$-closed in $B\left(H_{\iota} / K_{\iota}\right)$ by Lemma 2.3. Moreover, $G_{0} K_{\iota} / K_{\iota}$ is a cocompact connected normal subgroup of the connected Lie group $H_{\iota} / K_{\iota}$. Thus, by Corollary $2.9, B_{0}\left(G_{0} K_{\iota} / K_{\iota}\right)$ is $w^{*}$-closed in $B\left(G_{0} K_{\iota} / K_{\iota}\right)$. This proves that $B_{0}\left(G_{0} / G_{0} \cap K_{\iota}\right)$ is $w^{*}$-closed in $B\left(G_{0} / G_{0} \cap K_{\iota}\right)$.

We conclude this section with some remarks.

Remarks 2.11. (i) The connected reductive Lie groups with property $(T)$ and compact centres are precisely the groups of the form $G=\left(\mathbb{R}^{n} \times G_{1} \times \cdots \times G_{m}\right) / \Gamma$, where $G_{1}, \ldots, G_{m}$ are simple Lie groups not locally isomorphic to $\mathrm{SO}(k, 1), k \geq 2$, or $\mathrm{SU}(k, 1), k \geq 1$, and $\Gamma$ is a discrete cocompact subgroup of $\mathbb{R}^{n} \times Z_{1} \times \cdots \times Z_{m}$, the centre of $\mathbb{R}^{n} \times G_{1} \times \cdots \times G_{m}$.

Indeed, if $G$ is of this form, then $G / Z(G)=G_{1} \times \cdots \times G_{m}$, where $Z(G)$ denotes the centre of $G$, has property ( $T$ ) (see [14, Chap. 2, 13. Corollaire, 9. Remarque and Chap. 9]). As $Z(G)$ is compact, $G$ has property (T) [14, Chap. 1, 9. Proposition].

Conversely, let $G$ be a connected reductive Lie group with property $(T)$ and compact centre. Let $\widetilde{G}=\mathbb{R}^{n} \times G_{1} \times \cdots \times G_{m}$ be its universal covering group, where $G_{1}, \ldots, G_{m}$ are simple Lie groups with centres $Z_{1}, \ldots, Z_{m}$. The arguments used in the proof of Lemma 2.6 show that $G_{1}, \ldots, G_{m}$ have property $(T)$ and hence are not locally isomorphic to $\mathrm{SO}(k, 1)$ or $\mathrm{SU}(k, 1)$ (see [14, Chap. 6, 23. Corollaire]).

(ii) Let $G$ be a discrete group such that $B_{0}(G)$ is $w^{*}$-closed in $B(G)$. Then every element in $G$ has finite order. Indeed, this follows immediately by applying Lemma 2.2 to the cyclic subgroups of $G$.

(iii) Let $G$ be a linear group (that is, a subgroup $\mathrm{GL}(n, K)$ for some field $K$ ) with the discrete topology. If $G$ is infinite, then $B_{0}(G)$ is not $w^{*}$-closed in $B(G)$. In fact, this is clear from (ii) if $G$ has an element of infinite order. On the other 
hand, if $G$ is a torsion group, then it is well known to be locally finite [6, Theorem 9.2] and hence amenable, so that the claim follows from Lemma 2.2.

\section{Characterizations of compact groups}

If $G$ is a compact group then $A(G)=B(G)$. It seems likely that the converse is also true; i.e. $w^{*}$-closedness of $A(G)$ in $B(G)$ already forces $G$ to be compact. We have been able to show this for groups containing an almost connected open normal subgroup (Theorem 3.6). The case that remains open is that of a totally disconnected group.

We start with a lemma which will be generalized in Section 4 (Lemma 4.2).

Lemma 3.1. If $A(G)$ is $w^{*}$-closed in $B(G)$ and $H$ is an open subgroup of $G$, then $A(H)$ is $w^{*}$-closed in $B(H)$.

Proof. It suffices to show that the unit ball of $A(H)$ is $w^{*}$-closed in the unit ball of $B(H)$. Thus, let $\left(\varphi_{\iota}\right)$ be a net in $A(H)$ and $\varphi \in B(H)$ such that

$$
\left\|\varphi_{\iota}\right\| \leq 1,\|\varphi\| \leq 1 \text { and } \varphi_{\iota} \rightarrow \varphi
$$

in the $w^{*}$-topology. Let $\tilde{\varphi}_{\iota}$ and $\tilde{\varphi}$ denote the trivial extensions of $\varphi_{\iota}$ and $\varphi$ to $G$. Then $\tilde{\varphi}_{\iota} \in A(G),\left\|\tilde{\varphi}_{\iota}\right\| \leq 1$ and, for each $f \in L^{1}(G)$,

$$
\begin{aligned}
\int_{G} \tilde{\varphi}_{\iota}(x) f(x) d x=\int_{H} \varphi_{\iota}(h) f(h) d h \\
\quad \rightarrow \int_{H} \varphi(h) f(h) d h=\int_{G} \tilde{\varphi}(x) f(x) d x .
\end{aligned}
$$

Hence $\tilde{\varphi}_{\iota} \rightarrow \tilde{\varphi}$ in the $\sigma\left(B(G), L^{1}(G)\right)$-topology. Since $\left(\tilde{\varphi}_{\iota}\right)$ is a bounded net, it follows that $\left(\tilde{\varphi}_{\iota}\right)$ is $w^{*}$-convergent to $\tilde{\varphi}$. By hypothesis, $\tilde{\varphi} \in A(G)$ and so $\varphi \in$ $A(H)$.

Lemma 3.2. Let $K$ be a compact normal subgroup of $G$. If $A(G)$ is $w^{*}$-closed in $B(G)$, then $A(G / K)$ is $w^{*}$-closed in $B(G / K)$.

Proof. Consider the map $T_{K}: f \rightarrow T_{K} f$ from $L^{1}(G)$ onto $L^{1}(G / K)$ given by

$$
T_{K} f(x K)=\int_{K} f(x k) d k
$$

This map extends to a *-homomorphism from $C^{*}(G)$ onto $C^{*}(G / K)$ with dual map $T_{K}^{*}: B(G / K) \rightarrow B(G)$. Furthermore, $T_{K}^{*}(B(G / K))$ consists precisely of those functions in $B(G)$ that are constant on cosets of $K[8,(2.26)]$. Also, since $K$ is compact,

$$
T_{K}^{*}(A(G / K))=A(G) \cap T_{K}^{*}(B(G / K)) .
$$

Now, let $\varphi_{\iota} \in A(G / K)$ such that $\varphi_{\iota} \rightarrow \varphi$ in the $w^{*}$-topology for some $\varphi \in B(G / K)$. Then

$$
\left\langle T_{K}^{*}\left(\varphi_{\iota}\right), f\right\rangle=\left\langle\varphi_{\iota}, T_{K}(f)\right\rangle \rightarrow\left\langle\varphi, T_{K}(f)\right\rangle=\left\langle T_{K}^{*}(\varphi), f\right\rangle
$$

for each $f \in C^{*}(G)$. Thus, by hypothesis, $T_{K}^{*}(\varphi) \in A(G)$ and so

$$
T_{K}^{*}(\varphi) \in A(G) \cap T_{K}^{*}(B(G / K)),
$$

whence $\varphi \in A(G / K)$.

Lemma 3.3. Let $G$ be an almost connected locally compact group. If $A(G)$ is $w^{*}$ closed in $B(G)$, then $G$ is compact. 
Proof. Since an almost connected group is a projective limit of Lie groups and $A(G / K)$ is $w^{*}$-closed in $B(G / K)$ for every compact normal subgroup $K$ of $G$ (Lemma 3.2), we can assume that $G$ is a Lie group. Being a compactly generated Lie group, $G$ is second countable and hence $A(G)$ is a separable Banach space. By hypothesis,

$$
A(G)=B_{\lambda}(G)=C_{r}^{*}(G)^{*} .
$$

Now, a $C^{*}$-algebra $A$ with separable dual Banach space has a countable dual $\widehat{A}$ (see Section 1). It follows that $\widehat{G}_{r}$, the reduced dual of $G$, is countable. Finally, by $[2$, Theorem 2.5] a separable Lie group with countable reduced dual is compact. This shows that $G$ is compact.

Corollary 3.4. Let $G$ be any locally compact group and suppose that $A(G)$ is $w^{*}$ closed in $B(G)$. Then $G$ contains a compact open subgroup.

Proof. Since $G / G_{0}$ is totally disconnected, there exists an open subgroup $H$ of $G$ so that $H / G_{0}$ is compact. By Lemma 3.1, $A(H)$ is $w^{*}$-closed and hence $H$ is compact by Lemma 3.3 .

Lemma 3.5. If $G$ is a discrete group and $A(G)$ is $w^{*}$-closed in $B(G)$, then $G$ is finite.

Proof. Assume that $G$ is infinite. Then $G$ has a countable infinite subgroup $H$. By Lemma 3.1, $A(H)$ is $w^{*}$-closed in $B(H)$. As in the proof of Lemma 3.3 we now conclude that $\widehat{H}_{r}$ is countable. Applying Baggett's result again, it follows that $H$ is finite, a contradiction.

Theorem 3.6. Suppose that $G$ contains an almost connected open normal subgroup. Then $A(G)$ is $w^{*}$-closed in $B(G)$ if and only if $G$ is compact.

Proof. Let $N$ be an almost connected open normal subgroup of $G$. Then $A(N)$ is $w^{*}$-closed in $B(N)$ by Lemma 3.1, and Lemma 3.3 implies that $N$ is compact. By Lemma $3.2, A(G / N)$ is $w^{*}$-closed in $B(G / N)$. Since $N$ is open, Lemma 3.5 gives that $G / N$ is finite. Thus $G$ is compact.

We now turn to a second characterization of compact groups in terms of certain properties of the $w^{*}$-topology on $B(G)$. If $G$ is a compact group, then the $w^{*}$ topology and the norm topology agree on the unit sphere of $B(G)=A(G)[11$, Corollary 2]. We are going to establish the converse to this (see [22, Theorem 5] for the amenable case). Actually, we prove a stronger result in that we replace the unit sphere of $B(G)$ by the smaller set $P_{\lambda}(G)=B_{\lambda}(G) \cap P(G)$ of all normalized positive definite functions on $G$ associated to representations that are weakly contained in the left regular representation. Note that this property implies the Radon-Nikodym property for $B(G)$ but not conversely (see [11] and [33]).

For any locally compact group $G$, let $P_{\lambda}(G)=B_{\lambda}(G) \cap P(G)$, the set of all normalized continuous positive definite functions on $G$ associated to representations that are weakly contained in the left regular representation. $P_{\lambda}(G)$ is a $w^{*}$-compact convex subset of $B(G)$. We denote by $\operatorname{ex}\left(P_{\lambda}(G)\right)$ the set of extreme points of the $w^{*}$-compact convex subset $P_{\lambda}(G)$ of $B(G)$.

Lemma 3.7. Let $G$ be a locally compact group and $\varphi \in \operatorname{ex}\left(P_{\lambda}(G)\right)$. Suppose that $\varphi$ is a point of continuity of the identity map

$$
\left(\operatorname{ex}\left(P_{\lambda}(G)\right), w^{*}\right) \rightarrow\left(\operatorname{ex}\left(P_{\lambda}(G)\right),\|\cdot\|\right) .
$$

Then $\pi_{\varphi}$ is an isolated point in $\widehat{G}_{r}$. 
Proof. Notice first that $\operatorname{ex}\left(P_{\lambda}(G)\right) \subseteq \operatorname{ex}(P(G))$ because if $\varphi \in P_{\lambda}(G)$ and $\psi \in P(G)$ are such that $c \varphi-\psi$ is positive definite for some $c \geq 0$, then $\psi \in P_{\lambda}(G)$. By [5, 2.12.1], if $\varphi_{1}, \varphi_{2} \in \operatorname{ex}(P(G))$ and $\pi_{\varphi_{1}}$ and $\pi_{\varphi_{2}}$ are not equivalent, then $\left\|\varphi_{1}-\varphi_{2}\right\| \geq 2$. By assumption there exists a $w^{*}$-open subset $U$ of $\operatorname{ex}\left(P_{\lambda}(G)\right)$ such that

$$
U \subseteq\left\{\psi \in \operatorname{ex}\left(P_{\lambda}(G)\right):\|\psi-\varphi\|<2\right\} .
$$

It follows that $\pi_{\psi}=\pi_{\varphi}$ for all $\psi \in U$. Now, by [5, Theorem 3.4.11], the map $q: \psi \rightarrow \pi_{\psi}$ from $\operatorname{ex}\left(P_{\lambda}(G)\right)$ onto $\widehat{G}_{r}$ is open. Thus $\left\{\pi_{\varphi}\right\}=q(U)$ is open in $\widehat{G}_{r}$.

Lemma 3.8. Let $H$ be an open subgroup of $G$. If the identity map from $\left(P_{\lambda}(G), w^{*}\right)$ to $\left(P_{\lambda}(H),\|\cdot\|\right)$ is continuous, then the identity map from $\left(P_{\lambda}(H), w^{*}\right)$ to $\left(P_{\lambda}(H)\right.$, $\|\cdot\|)$ is continuous.

Proof. For any $\varphi \in P_{\lambda}(H)$, the trivial extension $\tilde{\varphi}$ belongs to $P_{\lambda}(G)$. Indeed, $\tilde{\varphi}$ is a positive definite function associated to the induced representation $\operatorname{ind}_{H}^{G} \pi_{\varphi}$, and $\pi_{\varphi} \prec \lambda_{H}$ implies

$$
\operatorname{ind}_{H}^{G} \pi_{\varphi} \prec \operatorname{ind}_{H}^{G} \lambda_{H}=\lambda_{G} .
$$

Let $\left(\varphi_{\alpha}\right)$ be a net in $P_{\lambda}(H)$ converging to $\varphi \in P_{\lambda}(H)$ in the $w^{*}$-topology. Then $\tilde{\varphi}_{\alpha} \rightarrow \tilde{\varphi}$ in the $w^{*}$-topology on $P_{\lambda}(G)$ (compare the proof of Lemma 3.1). By hypothesis, $\left\|\tilde{\varphi}_{\alpha}-\tilde{\varphi}\right\| \rightarrow 0$ and hence $\left\|\varphi_{\alpha}-\varphi\right\| \rightarrow 0$.

Theorem 3.9. For any locally compact group $G$ the following conditions are equivalent.

(i) $G$ is compact.

(ii) The $w^{*}$-topology and the norm topology agree on the unit sphere of $B(G)$.

(iii) The $w^{*}$-topology and the norm topology agree on $P_{\lambda}(G)$.

Proof. As mentioned above, (i) $\Rightarrow$ (ii) is due to Granirer and Leinert [11]. Since (ii) $\Rightarrow$ (iii) is trivial, it only remains to prove (iii) $\Rightarrow$ (i).

Assume that $G$ fails to be compact. Then $G$ contains a non-compact, $\sigma$-compact, open subgroup $H$. By Lemma 3.8, the $w^{*}$-topology and the norm topology coincide on $P_{\lambda}(H)$. It follows from Lemma 3.7 that $\widehat{H}_{r}$ is discrete. Since $H$ is $\sigma$-compact Theorem 7.6 of [34] now shows that $H$ is compact, a contradiction.

\section{When IS $A_{\gamma}(G) w^{*}$-CLOSED IN $B(G)$ ?}

For a locally compact group $G$ and any unitary representation $\pi$ of $G$, the Fourier space $A_{\pi}(G)$ associated to $\pi$ is defined to be the norm-closed linear subspace of $B(G)$ generated by all the coordinate functions of $\pi[1]$, that is, the functions of the form $x \rightarrow\langle\pi(x) \xi, \eta\rangle, \xi, \eta \in \mathcal{H}_{\pi}$.

The conjugation representation $\gamma_{G}$ (or simply $\gamma$, if no confusion can arise) on $L^{2}(G)$ is defined by

$$
\gamma_{G}(x) f(y)=\Delta(x)^{1 / 2} f\left(x^{-1} y x\right),
$$

$f \in L^{2}(G), x, y \in G$. The purpose of this section is to investigate the question of when $A_{\gamma}(G)$ is $w^{*}$-closed in $B(G)$. It will turn out that this is closely related to problems on the support of $\gamma$ as studied in [21]. We start with two simple facts on $A_{\pi}(G)$ for general representations $\pi$. 
Lemma 4.1. If $G$ is a compact group, then $A_{\pi}(G)$ is $w^{*}$-closed in $B(G)$ for every representation $\pi$ of $G$.

Proof. The $w^{*}$-closure $\overline{A_{\pi}(G)} w^{*}$ of $A_{\pi}(G)$ is the dual space of the $C^{*}$-algebra $\pi\left(C^{*}(G)\right)$, which is a quotient of $C^{*}(G)$. Hence each $\varphi \in \overline{A_{\pi}(G)} w^{*}$ is a linear combination of positive definite functions in $\overline{A_{\pi}(G)} w^{*}$. Therefore, it suffices to prove that every positive definite $\varphi \in{\overline{A_{\pi}(G)}}^{w^{*}}$ actually is in $A_{\pi}(G)$.

For that, notice that there is a net $\left(\varphi_{\alpha}\right)$ in $A_{\pi}(G)$ such that $\varphi_{\alpha} \rightarrow \varphi$ in the $w^{*}$ topology and $\left\|\varphi_{\alpha}\right\| \rightarrow\|\varphi\|$ (compare [10, p. 565]). By [11, Theorem A] it follows that $\left\|\varphi_{\alpha} \psi-\varphi \psi\right\| \rightarrow 0$ for every $\psi \in A(G)$. In particular, $\left\|\varphi_{\alpha}-\varphi\right\| \rightarrow 0$ by setting $\psi=1 \in B(G)=A(G)$. This shows that $\varphi \in A_{\pi}(G)$.

Lemma 4.2. Suppose that $\pi$ is a representation of $G$ such that $A_{\pi}(G)$ is $w^{*}$-closed in $B(G)$. Then $A_{\pi \mid H}(H)$ is $w^{*}$-closed in $B(H)$ for every open subgroup $H$ of $G$.

Proof. Recall that, by [1, Theorem 2.2], $\varphi \in B(G)$ belongs to $A_{\pi}(G)$ if and only if $\varphi$ can be written as

$$
\varphi=\sum_{n=1}^{\infty}\left\langle\pi(\cdot) \xi_{n}, \eta_{n}\right\rangle
$$

where $\xi_{n}, \eta_{n} \in \mathcal{H}_{\pi}$ and $\sum_{n=1}^{\infty}\left\|\xi_{n}\right\| \cdot\left\|\eta_{n}\right\|<\infty$. In particular, $A_{\pi \mid H}(H)=A_{\pi}(G) \mid H$. It suffices to show that the unit ball of $A_{\pi \mid H}(H)$ is $w^{*}$-closed in the unit ball of $B(H)$. Thus, let $\varphi_{i} \in A_{\pi \mid H}(H), i \in I$, and $\varphi \in B(H)$ such that $\left\|\varphi_{i}\right\| \leq 1,\|\varphi\| \leq 1$ and $\varphi_{i} \rightarrow \varphi$ in the $w^{*}$-topology. Choose representations

$$
\varphi_{i}=\sum_{n=1}^{\infty}\left\langle\pi(\cdot) \xi_{i n}, \eta_{i n}\right\rangle
$$

such that $\sum_{n=1}^{\infty}\left\|\xi_{\text {in }}\right\| \cdot\left\|\eta_{\text {in }}\right\| \leq 2$ (see [1, Proposition 2.9]). Define

$$
\psi_{i}(x)=\sum_{n=1}^{\infty}\left\langle\pi(x) \xi_{i n}, \eta_{i n}\right\rangle
$$

for all $x \in G$ and $i \in I$. Then $\psi_{i} \in A_{\pi}(G)$ and $\left\|\psi_{i}\right\| \leq 2$. Since the unit ball in $B(G)$ is $w^{*}$-compact, we can assume that $\psi_{i} \rightarrow \psi$ in the $w^{*}$-topology for some $\psi \in B(G)$. Now, $A_{\pi}(G)$ is $w^{*}$-closed in $B(G)$, so that $\psi \in A_{\pi}(G)$ and hence $\psi \mid H \in A_{\pi \mid H}(H)$. On the other hand, the restriction map $B(G) \rightarrow B(H)$ is $w^{*}$-continuous as $H$ is open (see Section 5). Indeed, this follows from the fact that $C^{*}(H)$ is a subalgebra of $C^{*}(G)$ whenever $H$ is open in $G$. Thus

$$
\varphi_{i}=\psi_{i}|H \rightarrow \psi| H \text { and } \varphi_{i} \rightarrow \varphi
$$

in the $w^{*}$-topology. This proves $\varphi=\psi \mid H \in A_{\pi \mid H}(H)$.

We now apply the preceding lemmas to the conjugation representation. The following corollary will be used several times in the sequel.

Corollary 4.3. Suppose that $G$ is second countable and $A_{\gamma_{G}}(G)$ is $w^{*}$-closed in $B(G)$. Then, for every open subgroup $H$ of $G$, supp $\gamma_{H}$ is countable.

Proof. Since $G$ is second countable, $A_{\gamma}(G)$ is norm separable. Since the restriction map from $B(G)$ to $B(H)$ is norm continuous, $A_{\gamma_{G} \mid H}(H)=A_{\gamma_{G}}(G) \mid H$ is norm 
separable. Now, since $\gamma_{H}$ is a subrepresentation of $\gamma_{G} \mid H$ and $A_{\gamma_{G} \mid H}(H)$ is $w^{*}$ closed in $B(H)$ by Lemma 4.2 ,

$$
\overline{A_{\gamma_{H}}(H)} w^{*} \subseteq A_{\gamma_{G} \mid H}(H)
$$

so that $\overline{A_{\gamma_{H}}(H)} w^{*}$ is norm separable. Thus $\gamma_{H}\left(C^{*}(H)\right)$ has a norm separable dual Banach space, $\overline{A_{\gamma_{H}}(H)} w^{*}$, and hence

$$
\operatorname{supp} \gamma_{H}=\gamma_{H}\left(C^{*}(H)\right)^{\wedge}
$$

is countable.

Corollary 4.4. Let $Z(G)$ denote the centre of $G$. If $G / Z(G)$ is compact, then $A_{\gamma_{G}}(G)$ is $w^{*}$-closed in $B(G)$.

Proof. For $z \in Z(G), \gamma_{G}(z)$ is the identity on $L^{2}(G)$. Thus $\pi(x Z(G))=\gamma_{G}(x)$, $x \in G$, defines a representation of $G / Z(G)$, and therefore $A_{\pi}(G / Z(G))$ is $w^{*}$ closed in $B(G / Z(G))$ by Lemma 4.1. Denoting by $q: G \rightarrow G / Z(G)$ the quotient homomorphism, we have

$$
A_{\gamma_{G}}(G)=A_{\pi}(G / Z(G)) \circ q .
$$

By $[1,(2.10)], A_{\gamma_{G}}(G)$ is $w^{*}$-closed in $B(G)$.

Our goal is to establish the converse to Corollary 4.4 for Lie groups with countably many connected components (Theorem 4.8). Apart from using various results from [21], a major step in proving the theorem will be the next lemma.

We remind the reader that a group $G$ is called an FC-group if all its conjugacy classes are finite. Such a group, more generally every locally compact group all of whose conjugacy classes are relatively compact, is amenable.

Lemma 4.5. Let $G$ be a countable discrete $\mathrm{FC}$-group. If supp $\gamma_{G}$ is countable, then $G$ has a finite commutator subgroup.

Proof. Let $S=\operatorname{supp} \gamma_{G}$ and notice first that points in $S$ are closed in $\widehat{G}$. Indeed, the primitive ideal space of any FC-group is a $T_{1}$ space (see [28, Theorem 5.2]) and $C_{\gamma}^{*}(G)$, being a separable $C^{*}$-algebra with countable dual, is of type I. Thus the points of $S$ are closed in $\widehat{G}$. Since $C^{*}(G)$ is unital, it follows that every $\sigma \in S$ is finite dimensional.

Next, employing the facts that points in $S$ are closed, that $S$ is countable and that duals of $C^{*}$-algebras are Baire spaces [5, (3.4.13)], a straightforward argument yields the existence of some dense subset $D$ of $S$ consisting of points that are also open in $S$.

Since $G$ is a countable amenable group,

$$
\bigcup_{\pi \in \widehat{G}} \operatorname{supp}(\pi \otimes \bar{\pi})
$$

is a dense subset of $S$ by [19, Theorem]. Let $\mathcal{C}(S)$ denote the set of all closed subsets of $S$, endowed with Fell's topology [10, p. 427]. By [18, Proposition 2], the mapping

$$
\pi \rightarrow \operatorname{supp}(\pi \otimes \bar{\pi}), \widehat{G} \rightarrow \mathcal{C}(S)
$$

is continuous. It follows that

$$
V=\{\pi \in \widehat{G}: \operatorname{supp}(\pi \otimes \bar{\pi}) \cap D \neq \varnothing\}
$$

is non-empty and open in $\widehat{G}$. 
Now, as points in $D$ are open in $S, \operatorname{supp}(\pi \otimes \bar{\pi})$ contains a finite dimensional subrepresentation for each $\pi \in V$. However, $\pi \otimes \bar{\pi}$ then also contains the trivial representation $1_{G}$. This can be seen as follows. Suppose that $\tau$ is finite dimensional and that $\tau \leq \pi \otimes \bar{\pi}$. Then

$$
1_{G} \leq \tau \otimes \bar{\tau} \leq \pi \otimes \overline{\pi \otimes \tau}
$$

and $\pi \otimes \tau$ is a (finite) direct sum of irreducible representations $\rho_{1}, \ldots, \rho_{n}$. Thus $1_{G} \leq \pi \otimes \overline{\rho_{i}}$ for some $i$, which is impossible unless $\rho_{i} \sim \pi$ (see [17, Proposition 2.4]). As is well-known, $1_{G} \leq \pi \otimes \bar{\pi}$ forces $\pi$ to be finite dimensional. Hence every $\pi \in V$ is finite dimensional.

Finally, for a discrete FC-group $G$, the existence of a non-empty open subset in $\widehat{G}$ consisting of finite dimensional representations implies that $G$ has a finite commutator subgroup. In fact, in this case the left regular representation of $G$ has a subrepresentation of type I, and then the commutator subgroup has to be finite by $[16$, Satz 1] (see also [32, Theorem 3]).

Lemma 4.6. Let $G$ be a locally compact group with open centre. Then, for each $a \in G$,

$$
\operatorname{ind}_{C(a)}^{G} 1_{C(a)} \prec \gamma_{G},
$$

where $C(a)$ denotes the centralizer of a in $G$.

Proof. Since $\operatorname{ind}_{C(a)}^{G} 1_{C(a)}$ is the cyclic representation of $G$ defined by the positive definite function $\chi_{C(a)}$, the characteristic function of $C(a)$, and since $C(a)$ is open, it suffices to show that given any compact subset $K$ of $G \backslash C(a)$, there is a positive definite function $\varphi$ associated to $\gamma_{G}$ such that $\varphi(x)=1$ for all $x \in C(a)$ and $\varphi(x)=0$ for all $x \in K$. Now, since

$$
C=\left\{a^{-1} x^{-1} a x: x \in K\right\}
$$

is compact and $e \notin C$, we find an open neighborhood $V$ of $e$, contained in the centre of $G$, such that $C V \cap V=\varnothing$. Let

$$
\varphi(x)=|V|^{-1}\left\langle\gamma_{G}(x) \chi_{a V}, \chi_{a V}\right\rangle
$$

for $x \in G$. Then it is easily verified that $\varphi(x)=1$ for all $x \in C(a)$ and $\varphi(x)=0$ for all $x \in K$.

Lemma 4.7. Suppose that $G$ is second countable and contains an open normal subgroup $N$ such that $G / N$ is abelian and every irreducible representation of $N$ is finite dimensional. If $\operatorname{supp} \gamma_{G}$ is countable, then every irreducible representation of $G$ is finite dimensional.

Proof. Given an irreducible representation $\pi$ of $G$, there exist a subgroup $H$ of $G$ and a finite dimensional irreducible representation $\tau$ of $H$ such that $N \subseteq H$ and

$$
\pi \sim \operatorname{ind}_{H}^{G} \tau .
$$

In fact, this has been shown in [7, Theorem 3.2.3] as an application of representation theory of crossed product $C^{*}$-algebras. We prefer to outline a direct argument for this within the framework of Mackey's unitary representation theory of group extensions [26].

Choose $\gamma \in \widehat{N}$ such that $\pi \mid N \sim G(\gamma)$, the $G$-orbit of $\gamma$ in $\widehat{N}$ under the action of $G$. Let $S$ denote the stability subgroup of $\gamma$. By [26, Theorems 8.2 and 8.3], there 
exist a multiplier $\omega$ on $S / N$, an irreducible $\omega$-representation $\rho$ of $S$ in $\mathcal{H}_{\gamma}$ and an irreducible $\omega$-representation $\sigma$ of $S / N$ such that

$$
\pi=\operatorname{ind}_{S}^{G}(\rho \otimes \sigma) .
$$

Now, an irreducible $\omega$-representation of an abelian group is weakly equivalent to the $\omega$-representation induced from some one-dimensional $\omega$-representation of a certain subgroup. Hence there exist a subgroup $H$ of $S$ containing $N$ and a one-dimensional $\omega$-representation $\lambda$ of $H / N$ such that

$$
\rho \otimes \sigma \sim \rho \otimes \operatorname{ind}_{H}^{S} \lambda=\operatorname{ind}_{H}^{S}(\rho \mid H \otimes \lambda) .
$$

Let $\pi=\rho \mid H \otimes \lambda$, a finite dimensional ordinary representation. Then

$$
\pi \sim \operatorname{ind}_{S}^{G}\left(\operatorname{ind}_{H}^{S}(\rho \mid H \otimes \lambda)\right)=\operatorname{ind}_{H}^{G} \tau
$$

as required.

It follows that

$$
\begin{aligned}
\pi \otimes \bar{\pi} & =\operatorname{ind}_{H}^{G}(\tau \otimes \bar{\pi} \mid H) \succ \operatorname{ind}_{H}^{G}(\tau \otimes \bar{\tau}) \\
& \succ \operatorname{ind}_{H}^{G} 1_{H} \sim \widehat{G / H} .
\end{aligned}
$$

On the other hand, $\pi \otimes \bar{\pi}$ is weakly contained in $\gamma_{G}$ since $G$ is amenable. Now, as supp $\gamma_{G}$ is countable and $H$ is open and $G / H$ is abelian, $H$ must have finite index in $G$. Thus, $\operatorname{ind}_{H}^{G} \tau$ is finite dimensional and hence so is $\pi$.

Theorem 4.8. Suppose that $G$ is a Lie group with countably many connected components. If $A_{\gamma_{G}}(G)$ is $w^{*}$-closed in $B(G)$, then $G / Z(G)$ is compact.

Proof. For any normal subgroup $N$ of $G$, let $q_{N}: G \rightarrow G / N$ denote the quotient homomorphism. Since $A_{\gamma_{G}}(G)$ is $w^{*}$-closed in $B(G)$ and $G$ is second countable, and since the connected component $G_{0}$ of $e$ is open, $\operatorname{supp} \gamma_{G_{0}}$ is countable by Corollary 4.3. Theorem 3.4 of [21] yields that $G_{0}=V \times C$, the direct product of a vector group $V$ and a compact group $C$. Clearly, $C$ is normal in $G$.

We claim that $V=G_{0} / C$ is contained in the centre of $G / C$. To that end, fix $a \in G$ and consider the open subgroup $H$ of $G$ generated by $a$ and $G_{0}$. Then $\operatorname{supp} \gamma_{H}$ is countable (Corollary 4.3), and since

$$
\gamma_{G / C} \circ q_{C} \prec \gamma_{H}
$$

due to the compactness of $C\left[19\right.$, Remark 1], it follows that $\gamma_{H / C}$ has a countable support. Since $G_{0} / C$ is a vector group and $(H / C) /\left(G_{0} / C\right)=H / G_{0}$ is abelian, an application of Lemma 3.2 in [21] shows that $G_{0} / C$ is in the centre of $H / C$. Since $a$ is arbitrary, $G_{0} / C$ is central in $G / C$.

Next we are going to prove that $G / G_{0}$ has a finite commutator subgroup. Passing to $F=G / C$, we know that $\gamma_{F}$ has countable support, and since $V$ is central in $F$, by [21, Lemma 1.1]

$$
\gamma_{F / V} \circ q_{V} \prec \gamma_{F},
$$

so that $\operatorname{supp} \gamma_{F / V}$ is countable. Let $D=F / V$ and denote by $D_{f}$ the finite conjugacy class subgroup of $D$. Then, by [20, Theorem 1.8]

$$
\lambda_{D / D_{f}} \circ q_{D_{f}} \prec \gamma_{D}
$$

where $\lambda_{D / D_{f}}$ is the regular representation of $D / D_{f}$. Thus the reduced dual of $D / D_{f}$ is countable, and hence $D / D_{f}$ is finite (see $[2,34]$ ). At this stage we know in particular that $G$ is amenable, so that $\gamma_{G / N} \circ q_{N} \prec \gamma_{G}$ for each closed normal 
subgroup $N$ of $G$ [21, Lemma 1.1]. Also, $\gamma_{D_{f}}$ has countable support and therefore the commutator subgroup $D_{f}^{\prime}$ of $D_{f}$ is finite by Lemma 4.5. Let $N$ be the inverse image of $D_{f}^{\prime}$ in $G$ and let $E=G / N$. Then $\gamma_{E}$ has countable support, and $E$ possesses an abelian normal subgroup $A$ of finite index, namely $A=D_{f} / D_{f}^{\prime}$. We apply Lemma 4.6 to $E$ and obtain that

$$
\operatorname{ind}_{C(x)}^{E} 1_{C(x)} \prec \gamma_{E}
$$

for every $x \in E$. It follows that

$$
\operatorname{ind}_{C(x) \cap A}^{A} 1_{C(x) \cap A} \prec\left(\operatorname{ind}_{C(x)}^{E} 1_{C(x)}\right)\left|A \prec \gamma_{E}\right| A,
$$

and $\gamma_{E} \mid A$ has countable support since $\gamma_{E}$ does and $E / A$ is finite. However, $A$ being abelian, countability of

$$
(A / C(x) \cap A)^{\wedge}=\operatorname{supp}\left(\operatorname{ind}_{C(x) \cap A}^{A} 1_{C(x) \cap A}\right)
$$

implies that $C(x) \cap A$ is of finite index in $A$. Hence $C(x)$ has finite index in $E$ for every $x \in E$. Therefore $G / N$ is an FC-group, and Lemma 4.5 implies that $E$ has a finite commutator subgroup. Recalling that $N / G_{0}=D_{f}^{\prime}$ is finite, we conclude that $G / G_{0}$ has a finite commutator subgroup.

Since $G / G_{0}$ has a finite commutator subgroup, there exists a normal subgroup $N$ of $G$ such that $G_{0} \subseteq N, N / G_{0}$ is finite and $G / N$ is abelian. Now, $G_{0}=V \times C$, and this implies that all the irreducible representations of $N$ are finite dimensional. Since supp $\gamma_{G}$ is countable, Lemma 4.7 shows that $G$ has only finite dimensional irreducible representations.

On the other hand, $G$ has a relatively compact commutator subgroup. To see this, notice first that since $V$ is contained in the centre of $N / C, N / C$ has a finite commutator subgroup. Denoting its inverse in $G$ by $K, N / K$ is isomorphic to $V$. By arguments that have previously been used, $V$ is central in $G / K$, and applying Lemma 4.6 again, this time to $G / K$, we conclude that $G / K$ is a group with finite conjugacy classes.

Thus $G$ is a group with relatively compact conjugacy classes all of whose irreducible representations are finite dimensional. Since, in addition, $G$ is a Lie group, combining Theorem 2 of [27] and Lemma 5.4 of [24] shows that $G / Z(G)$ is compact.

\section{5. $w^{*}$-CONTINUITY OF THE RESTRICTION MAP}

Let $G$ be a locally compact group, and let $H$ be a closed subgroup of $G$. In this section we study the question of when the restriction map

$$
\Phi: B(G) \rightarrow B(H), \varphi \rightarrow \varphi \mid H
$$

is continuous for the $w^{*}$-topologies. Clearly, if $H$ is open then $C^{*}(H)$ is a subalgebra of $C^{*}(G)$ and hence $\Phi$ is $w^{*}$-continuous. We are going to establish the following converse.

Theorem 5.1. Let $H$ be a closed subgroup of the locally compact group $G$. If the restriction map $B(G) \rightarrow B(H)$ is continuous for the $w^{*}$ - topologies, then $H$ is open in $G$.

Let $M(G)$ denote the algebra of all bounded measures on $G$. The canonical embedding of $L^{1}(G)$ into $M(G)$ extends to an isometric *-homomorphism of $C^{*}(G)$ 
into $C^{*}(M(G))$, the enveloping $C^{*}$-algebra of $M(G)$. Therefore we may (and shall) identify $C^{*}(G)$ with a closed two-sided ideal of $C^{*}(M(G))$.

Let $H$ be a closed subgroup of $G$. For $f \in L^{1}(H)$, let $\mu_{f} \in M(G)$ denote the measure on $G$ defined by $f$. The mapping $f \rightarrow \mu_{f}$ from $L^{1}(H)$ into $M(G)$ extends to a *-homomorphism $\Psi: C^{*}(H) \rightarrow C^{*}(M(G))$. By general principles, the restriction map $\Phi: B(G) \rightarrow B(H)$ is $w^{*}$-continuous if and only if $\Phi$ is the transpose of some continuous linear mapping $\Theta: C^{*}(H) \rightarrow C^{*}(G)$. It is easy to verify that, in this case, $\Theta$ has to agree with $\Psi$ on $L^{1}(H)$. Hence we have the following lemma.

Lemma 5.2. The restriction map $\Phi: B(G) \rightarrow B(H)$ is $w^{*}$-continuous if and only if the range of $\Psi: C^{*}(H) \rightarrow C^{*}(M(G))$ is contained in $C^{*}(G)$.

Remark 5.3. It is interesting to notice that the homomorphism $\Psi: C^{*}(H) \rightarrow$ $C^{*}(M(G))$ need not always be injective (see [4]).

Now, let $\rho$ denote the right regular representation of $G$ on $L^{2}(G)$ and $C_{r}^{*}(M(G))$ the $C^{*}$-subalgebra of $\mathcal{L}\left(L^{2}(G)\right)$ generated by the set of all operators $\rho(\mu), \mu \in$ $M(G)$. The mapping $f \rightarrow \mu_{f}$ from $L^{1}(H)$ into $M(G)$ extends to a ${ }^{*}$-homomorphism

$$
\Psi_{r}: C^{*}(H) \rightarrow C_{r}^{*}(M(G)) .
$$

It is clear that $\Psi_{r}$ is the composition of $\Psi$ and the canonical homomorphism from $C^{*}(M(G))$ onto $C_{r}^{*}(M(G))$.

In view of Lemma 5.2, we thus observe that Theorem 5.1 will be a consequence of the following stronger result.

Theorem 5.4. Let $H$ be a closed subgroup of the locally compact group $G$. If the range of the homomorphism $\Psi_{r}: C^{*}(H) \rightarrow C_{r}^{*}(M(G))$ is contained in $C_{r}^{*}(G)$, then $H$ is open in $G$.

The proof of Theorem 5.4 depends on two elementary lemmas, the first of which appears also in [25]. For the sake of completeness, however, we give a very short and different proof.

Lemma 5.5. Let $G$ be a locally compact group and let $T \in C_{r}^{*}(G)$. Suppose that $K$ is a compact subset of $G$ and regard $L^{2}(K)$ as a closed subspace of $L^{2}(G)$ in the usual manner. Then the restriction

$$
T \mid L^{2}(K): L^{2}(K) \rightarrow L^{2}(G)
$$

of $T$ to $L^{2}(K)$ is a compact operator.

Proof. Of course, it suffices to prove the statement for operators $T$ of the form $T=\rho(f)$ where $f \in C_{c}(G)$. Let $K^{\prime}=\operatorname{supp} f \cdot K$. Then $T g \in L^{2}\left(K^{\prime}\right)$ for all $g \in L^{2}(K)$. Choose $f_{j} \in C_{c}\left(K^{\prime}\right)$ and $g_{j} \in C_{c}(K)$ such that, as $n \rightarrow \infty$,

$$
\int_{G} \int_{G}\left|\sum_{j=1}^{n} f_{j}(x) g_{j}(y)-f(x y)\right|^{2} d x d y \rightarrow 0 .
$$

It is straightforward to verify that this implies that $T: L^{2}(K) \rightarrow L^{2}\left(K^{\prime}\right)$ is a norm limit of finite rank operators.

Lemma 5.6. Let $(X, \mu)$ be a probability measure space without atoms. Then the canonical embedding $L^{2}(X) \rightarrow L^{1}(X)$ fails to be compact. 
Proof. It suffices to show that there is an orthonormal sequence $\left(f_{n}\right)_{n}$ in $L^{2}(X)$ such that $\left\|f_{n}\right\|_{\infty} \leq 1$. Indeed, we then have

$$
2=\left\|f_{n}-f_{m}\right\|_{2}^{2}=\int_{X}\left|f_{n}(x)-f_{m}(x)\right|^{2} d \mu(x) \leq\left\|f_{n}-f_{m}\right\|_{1}
$$

for all $n, m \in \mathbb{N}, n \neq m$, and hence no subsequence of $\left(f_{n}\right)_{n}$ can converge in $L^{1}(X)$.

Since $\mu$ has no atoms, there exists, for every $0 \leq r \leq 1$, a measurable subset $A$ of $X$ with $\mu(A)=r$ (see [13, Section 4.1, Exercise 1]). Therefore, we can choose inductively, for every $n \in \mathbb{N}$, disjoint measurable subsets

$$
A_{1}^{(n)}, A_{2}^{(n)}, \ldots, A_{2^{n-1}}^{(n)}
$$

of $X$ with the following properties:

(1) $\mu\left(A_{i}^{(n)}\right)=\frac{1}{2^{n-1}}$ for $i=1, \ldots, 2^{n-1}$.

(2) $A_{2 i-1}^{(n+1)}, A_{2 i}^{(n+1)} \subseteq A_{i}^{(n)}$ for $i=1, \ldots, 2^{n-1}$.

For each $n \in \mathbb{N}$, define a function $f_{n} \in L^{2}(X)$ (a kind of Rademacher function) by setting

$$
f_{n}(x)=(-1)^{i} \text { for } x \in A_{i}^{(n)} \text { and } i=1, \ldots, 2^{n-1} .
$$

By construction and (1) and (2), we obviously have

$$
\left\|f_{n}\right\|_{\infty}=\left\|f_{n}\right\|_{2}=1 \text { and } \int_{X} f_{n}(x) f_{m}(x) d \mu(x)=0, \quad n \neq m .
$$

This completes the proof.

Proof of Theorem 5.4. Let $H$ be a closed subgroup of $G$ and suppose $\Psi_{r}\left(C^{*}(H)\right)$ is contained in $C_{r}^{*}(G)$. Fix a Bruhat function $\beta$ on $G$ for $H$ (see [31, Chapter 8]), that is, a non-negative continuous function $\beta$ on $G$ with the following properties:

(i) For every compact subset $K$ of $G, \beta$ agrees on $K H$ with the restriction of some function from $C_{c}(G)$.

(ii) $\int_{H} \beta(x h) d h=1$ for all $x \in G$.

Let $\Delta_{G}$ and $\Delta_{H}$ denote the modular functions of $G$ and $H$, respectively, and define a function $q$ on $G$ by

$$
q(x)=\int_{H} \beta(x h) \Delta_{G}(h) \Delta_{H}(h)^{-1} d h .
$$

Since $q(x h)=q(x) \Delta_{G}(h)^{-1} \Delta_{H}(h)$ for all $x \in G$ and $h \in H$, there exists a quasiinvariant measure $d_{q} \dot{x}$ on $\dot{G}=G / H$ such that

$$
\int_{G}\left(\frac{f(x h)}{q(x h)} d h\right) d_{q} \dot{x}=\int_{G} f(x) d x
$$

for all $f \in L^{1}(G)$ [31, Chapter 8].

Now let $\dot{K} \subseteq \dot{G}$ be a compact neighbourhood of $\dot{e}$ in $\dot{G}$. We are going to prove that $\dot{K}$ is finite. Clearly, this will imply that $H$ is open. Let $\pi: G \rightarrow \dot{G}$ denote the quotient map and choose a compact neighbourhood $K$ of $e$ in $G$ so that $\pi(K)=\dot{K}$. Fix a continuous function $f$ with compact support on $H$ such that

$$
\int_{H} f(h) \Delta_{G}(h)^{-1} d h \neq 0 .
$$


Let $K^{\prime}=K H \cap \operatorname{supp} \beta$, which is a compact subset of $G$. By Lemma 5.5, the restriction of $\rho\left(\mu_{f}\right)$, the right convolution operator defined by $\mu_{f}$, to $L^{2}\left(K^{\prime}\right)$ is a compact operator. Define a linear mapping

$$
T: L^{2}\left(\dot{K}, d_{q} \dot{x}\right) \rightarrow L^{2}\left(K^{\prime}\right)
$$

by $T \dot{g}(x)=\dot{g}(\dot{x}) \beta(x)^{1 / 2}$ for $\dot{g} \in L^{2}\left(\dot{K}, d_{q} \dot{x}\right)$ and $x \in K$. Since

$$
\int_{H} \frac{\beta(x h)}{q(x h)} d h=\frac{1}{q(x)} \int_{H} \beta(x h) \Delta_{G}(h) \Delta_{H}(h)^{-1} d h=1
$$

for all $x \in G$, we have

$$
\begin{aligned}
\|T \dot{g}\|_{2}^{2} & =\int_{\dot{G}}\left(\int_{H} \frac{|\dot{g}(\dot{x})|^{2} \beta(x h)}{q(x h)} d h\right) d_{q} \dot{x} \\
& =\int_{\dot{G}}|\dot{g}(\dot{x})|^{2} d_{q} \dot{x}=\|\dot{g}\|_{2}^{2} .
\end{aligned}
$$

Thus $T$ is a bounded linear operator. Set

$$
q_{1}(x)=\int_{H} \beta(x h)^{1 / 2} \Delta_{G}(h) \Delta_{H}(h)^{-1} d h
$$

for $x \in G$. Then, for all $x \in G$ and $h \in H$,

$$
q_{1}(x h)=q_{1}(x) \Delta_{G}(h)^{-1} \Delta_{H}(h) .
$$

Hence there exists a quasi-invariant measure $d_{q_{1}} \dot{x}$ on $\dot{G}$ such that

$$
\int_{\dot{G}}\left(\int_{H} \frac{q(x h)}{q_{1}(x h)} d h\right) d_{q_{1}} \dot{x}=\int_{G} g(x) d x
$$

for all $g \in L^{1}(G)$. Let

$$
S: L^{1}(G) \rightarrow L^{1}\left(\dot{G}, d_{q_{1}} \dot{x}\right)
$$

be the linear operator defined by

$$
S g(\dot{x})=\int_{H} \frac{g(x h)}{q_{1}(x h)} d h
$$

for $g \in C_{c}(G) . S$ is bounded since

$$
\|S g\|_{L^{1}\left(\dot{G}, d_{q_{1}} \dot{x}\right)} \leq \int_{\dot{G}}\left(\int_{H} \frac{|g(x h)|}{q_{1}(x h)} d h\right) d_{q_{1}} \dot{x}=\|g\|_{1} .
$$

Observe next that $\rho\left(\mu_{f}\right)$ maps $L^{2}\left(K^{\prime}\right)$ into $L^{2}\left(K^{\prime \prime}\right)$ for some compact subset $K^{\prime \prime}$ of $G$. Hence $\rho\left(\mu_{f}\right) T$ map $L^{2}\left(\dot{K}, d_{q} \dot{x}\right)$ into $L^{1}(G)$, and we may consider the bounded linear operator

$$
S \rho\left(\mu_{f}\right) T: L^{2}\left(\dot{K}, d_{q} \dot{x}\right) \rightarrow L^{1}\left(\dot{G}, d_{q_{1}} \dot{x}\right) .
$$

For $\dot{g} \in C(\dot{K})$, we have

$$
\begin{aligned}
S \rho\left(\mu_{f \mid H}\right) T(\dot{g})(\dot{x}) & =\dot{g}(\dot{x}) \int_{H} \frac{1}{q_{1}(x h)}\left(\int_{H} \beta(x h k)^{1 / 2} f(k) d k\right) d h \\
& =\dot{g}(\dot{x}) \int_{H} f(k)\left(\int_{H} \frac{\beta(x h k)^{1 / 2}}{q_{1}(x h)} d h\right) d k .
\end{aligned}
$$


However, for $x \in G$ and $k \in K$,

$$
\begin{aligned}
\int_{H} \frac{\beta(x h k)^{1 / 2}}{q_{1}(x h)} d h & =\Delta_{H}(k)^{-1} \int_{H} \frac{\beta(x h)^{1 / 2}}{q_{1}\left(x h k^{-1}\right)} d h \\
& =\Delta_{G}(k)^{-1} \int_{H} \frac{\beta(x h)^{1 / 2}}{q(x h)} d h=\Delta_{G}(k)^{-1} .
\end{aligned}
$$

Thus, setting $\alpha=\int_{H} f(k) \Delta_{G}(k)^{-1} d k \neq 0$, we obtain that

$$
S \rho\left(\mu_{f \mid H}\right) T(\dot{g})=\alpha \dot{g}
$$

for all $\dot{g} \in L^{2}\left(\dot{K}, d_{q} \dot{x}\right)$. Now, by hypothesis and Lemma 5.5 , the restriction of $\rho\left(\mu_{f \mid H}\right)$ to $L^{2}\left(K^{\prime}\right)$ is compact. Hence

$$
\alpha I: L^{2}\left(\dot{K}, d_{q} \dot{x}\right) \rightarrow L^{1}\left(\dot{K}, d_{q_{1}} \dot{x}\right)
$$

is a compact operator.

Finally, notice that since

$$
d_{q_{1}} \dot{x}=\frac{q_{1}(x)}{q(x)} d_{q} \dot{x}
$$

and $\frac{q_{1}}{q}$ is a strictly positive continuous function on the compact set $\dot{K}$, the corresponding $L^{1}$-spaces are equal and the $L^{1}$-norms are equivalent. Hence the embedding

$$
L^{2}\left(\dot{K}, d_{q} \dot{x}\right) \rightarrow L^{1}\left(\dot{K}, d_{q} \dot{x}\right)
$$

is compact. By Lemma $5.6, \dot{K}$ has to have atoms. However, this implies that $\dot{K}$ is finite.

\section{REFERENCES}

1. G. Arsac, Sur l'espace de Banach engendré par les coefficients d'une représentation unitaire, Publ. Dép. Math. Lyon 13 (1976), 1-101. MR 56:3180

2. L. Baggett, A separable group having a discrete dual space is compact, J. Funct. Anal. 10 (1972), 131-148. MR 49:10816

3. M. B. Bekka, A. T. Lau, and G. Schlichting, On invariant subalgebras of the Fourier-Stieltjes algebra of a locally compact group, Math. Ann. 294 (1992), 513-522. MR 93k:43606

4. M. B. Bekka and A. Valette, Lattices in semisimple Lie groups and multipliers of group $C^{*}$-algebras, Astérisque, no. 232, Soc. Math. France, 1995, pp. 67-79. MR 97a:22015

5. J. Dixmier, $C^{*}$-algebras, Amsterdam, 1977. MR 56:16388

6. J. D. Dixon, The structure of linear groups, London, 1971.

7. S. Echterhoff, Crossed products with continuous trace, Memoirs Amer. Math. Soc. 123 (1996), no. 586. CMP 97:03

8. P. Eymard, L'algèbre de Fourier d'un groupe localement compact, Bull. Soc. Math. France 92 (1964), 181-236. MR 37:4208

9. J. M. G. Fell, Weak containment and induced representations. II, Trans. Amer. Math. Soc. 110 (1964), 424-447. MR 28:3114

10. J. M. G. Fell and R. S. Doran, Representations of *-algebras, locally compact groups and Banach *-algebraic bundles, Vol. I, II, Boston, 1988. MR 90c:46001; MR 90c: 46002

11. E. Granirer and M. Leinert, On some topologies which coincide on the unit sphere of the Fourier-Stieltjes algebra $B(G)$ and of the measure algebra $M(G)$, Rocky Mountain J. Math. 11 (1981), 459-472. MR 85f:43009

12. F. P. Greenleaf, Amenable actions of locally compact groups, J. Funct. Anal. 4 (1969), 295315. MR 40:268

13. P. R. Halmos, Measure theory, New York, 1950. MR 11:504d

14. P. de la Harpe and A. Valette, La propriété $(T)$ de Kazhdan pour les groupes localement compacts, Astérisque 175, Soc. Math. France, 1989. MR 90m:22001 
15. H. E. Jensen, Scattered $C^{*}$-algebras, Math. Scand. 41 (1977), 308-314. MR 58:2321

16. E. Kaniuth, Der Typ der regulären Darstellung diskreter Gruppen, Math. Ann. 182 (1969), 334-339. MR 41:5516

17. E. Kaniuth, Weak containment and tensor products of group representations. II, Math. Ann. 270 (1985), 1-15. MR 86j:22004

18. E. Kaniuth, On topological Frobenius reciprocity for locally compact groups, Arch. Math. 48 (1987), 286-297. MR 89d:22082

19. E. Kaniuth, On the conjugation representation of a locally compact group, Math. Z. 202 (1989), 275-288. MR 90k:22003

20. E. Kaniuth and A. Markfort, The conjugation representation and inner amenability of discrete groups, J. Reine Angew. Math. 432 (1992), 23-37. MR 93m:22006

21. E. Kaniuth and A. Markfort, Locally compact groups whose conjugation representations satisfy a Kazhdan type property or have countable support, Math. Proc. Camb. Phil. Soc. 116 (1994), 79-97. MR 95d:22006

22. A. T. Lau and P. Mah, Normal structure in dual Banach spaces associated to locally compact groups, Trans. Amer. Math. Soc. 310 (1988), 341-353. MR 89e:43004

23. A. T. Lau and A. Ülger, Some geometric properties of the Fourier and Fourier Stieltjes algebras of locally compact groups, Arens regularity and related problems, Trans. Amer. Math. Soc. 337 (1993), 321-359. MR 93g:22007

24. J. R. Liukkonen, Dual spaces of groups with precompact conjugacy classes, Trans. Amer. Math. Soc. 180 (1973), 85-108. MR 47:6937

25. V. Losert, Properties of the Fourier algebra that are equivalent to amenability, Proc. Amer. Math. Soc. 92 (1984), 347-353. MR 86b:43010

26. G. W. Mackey, Unitary representations of group extensions, Acta Math. 99 (1958), 265-311. MR 20:4789

27. C. C. Moore, Groups with finite dimensional irreducible representations, Trans. Amer. Math. Soc. 166 (1972), 401-410. MR 46:1960

28. R. D. Mosak, The $L^{1}$ - and $C^{*}$-algebras of $[F I A]_{B}^{-}$groups and their representations, Trans. Amer. Math. Soc. 163 (1972), 277-310. MR 45:2096

29. A. L. T. Paterson, Amenability, Providence, Rhode Island, 1988. MR 90e:43001

30. J. P. Pier, Amenable locally compact groups, New York, 1984. MR 86a:43001

31. H. Reiter, Classical harmonic analysis and locally compact groups, Oxford, 1968. MR 46:5933

32. K. F. Taylor, The type structure of the regular representation of a locally compact group, Math. Ann. 222 (1976), 211-224. MR 54:12965

33. K. F. Taylor, Geometry of the Fourier algebras and locally compact groups with atomic unitary representations, Math. Ann. 262 (1983), 183-190. MR 84k:43020

34. S. P. Wang, On isolated points in the dual spaces of locally compact groups, Math. Ann. 218 (1975), 19-34. MR 52:5863

35. R. J. Zimmer, Ergodic theory and semisimple groups, Boston/Basel/New York, 1984. MR $\mathbf{8 6 j}: 22014$

Département de Mathématiques, Université de Metz, F - 57045 Metz, France

E-mail address: bekka@poncelet.univ-metz.fr

Fachbereich Mathematik/Informatik, Universität Paderborn, D - 33095 Paderborn, GERMANY

E-mail address: kaniuth@uni-paderborn.de

Department of Mathematical Sciences, University of Alberta, Edmonton, Canada T6G 2G1

E-mail address: tlau@vega.math.ualberta.ca

Mathematisches Institut, Technische Universität München, D - 80290 München, GerMANY

E-mail address: gschlich@mathematik.tu-muenchen.de 